Article

\title{
Characterization of Hydrothermal Deposition of Copper Oxide Nanoleaves on Never-Dried Bacterial Cellulose
}

\author{
W. Ross Warren ${ }^{(D)}$ and Dennis R. LaJeunesse * \\ Department of Nanoscience, Joint School of Nanoscience and Nanoengineering, University of North Carolina \\ Greensboro, Greensboro, NC 27401, USA; wrwarren@uncg.edu \\ * Correspondence: drlajeun@uncg.edu; Tel.: +1(336)-285-2866
}

Received: 27 August 2019; Accepted: 25 October 2019; Published: 27 October 2019

check for updates

\begin{abstract}
Bacterial cellulose (BC) has attracted a great deal of interest due to its green synthesis and biocompatibility. The nanoscale dimension of $\mathrm{BC}$ nanofibers generates an enormous surface area that enhances interactions with water and soluble components within aqueous solution. Recent work has demonstrated that $\mathrm{BC}$ is a versatile platform for the formation of metal/metal oxide nanocomposites. Copper oxide $(\mathrm{CuO})$ is a useful material to compare nanomaterial deposition on $\mathrm{BC}$ with other cellulosic materials because of copper's colorimetric reaction as it forms copper hydroxide $\left(\mathrm{Cu}(\mathrm{OH})_{2}\right)$ and transitions to $\mathrm{CuO}$. In this research, we found that never-dried $\mathrm{BC}$ readily deposits $\mathrm{CuO}$ into its matrix in a way that does not occur on cotton, dried BC, or regenerated cellulose fibers. We conclude that hydroxyl group availability does not adequately explain our results and that intrafibrillar pores in never-dried $\mathrm{BC}$ nanofibers play a critical role in $\mathrm{CuO}$ deposition.
\end{abstract}

Keywords: bacterial cellulose; nanoparticle synthesis; nanocomposite; biomaterial

\section{Introduction}

Cellulose is a linear chain polysaccharide that is composed of $\beta(1 \rightarrow 4)$ linked D-glucose subunits and is the most abundant biopolymer on earth [1]. Nanocellulose materials are a collection of nanomaterials that include nanofibrillated cellulose (NFC), nanocrystalline cellulose (NCC), and bacterial cellulose (BC) [2-4]. The nanoscale dimensions of cellulose in these materials generates an enormous surface area that enhances interactions with water and soluble components within aqueous solution [5]. Nanoscale cellulose has attracted a great deal of interest due to its biodegradability, mechanical properties, and sustainability [4,5]. BC is a highly pure and crystalline cellulosic material that is secreted from certain bacteria (i.e., Gluconacetobacter hansenii); each bacterium produces a single nanocellulose fiber which entangles together with other nearby fibers to create a gel-like non-woven mat called a pellicle [3,5,6]. Purified BC pellicles are transparent and durable materials that are composed of a nonwoven mat of cellulose ribbons; each BC nanoribbon is 40-60 nm wide, $10-20 \mathrm{~nm}$ tall, and has a crystal structure that is predominately cellulose I $\alpha$ [7-9]. In applied nanocomposite materials, BC has been used for supercapacitors, ion detection, $\mathrm{pH}$ sensing, and glucose sensing [10-12].

Recently, BC has been a highly active area of research as a synthetic platform for the generation of metal and metal oxide nanocomposites. A recent and extremely comprehensive review has shown that $\mathrm{BC}$ functions as the foundation for BC-metal oxide nanocomposites as well as a range of BC-metal sulfide and BC-metal nanocomposites [13]. Both solvo- and hydro-thermal processes have been used to deposit a variety of metal oxide (e.g., $\mathrm{ZnO}, \mathrm{TiO}_{2}$, and $\mathrm{Fe}_{3} \mathrm{O}_{4}$.) nanomaterials into the $\mathrm{BC}$ matrix [14-17]. In these examples, the $\mathrm{BC}$ nanocomposite is chemically stable with the nanomaterial incorporated throughout the $\mathrm{BC}$ matrix. More recent work describes a hydrothermal process for making a $\mathrm{BC}-\mathrm{CuO}$ nanocomposite [18]. 
Although recent work has demonstrated that $\mathrm{BC}$ is an extremely versatile substrate for the generation of metal/metal oxide nanocomposite materials, little work has been done to describe the properties of $B C$ that enable these synthetic reactions [13]. Much of the work that describes deposition of metal/metal oxide nanomaterials into $\mathrm{BC}$ matrixes suggests that there is an interaction between the solubilized metal ion and the hydroxyl groups of cellulose which facilitates nanomaterial deposition. In this paper, we use $\mathrm{CuO}$ as a model nanomaterial to compare nanomaterial deposition on $\mathrm{BC}$ with other cellulosic materials due to its distinct XRD spectrum and colorimetric products. Furthermore, $\mathrm{CuO}$ and other copper materials have significant roles in many catalytic, energy, and semiconductor applications $[19,20]$. In these experiments we found that $\mathrm{BC}$ mediated deposition of $\mathrm{CuO}$ resulted in the formation of $\mathrm{CuO}$ nanoleaves under reaction condition that were far milder and more environmentally friendly than other $\mathrm{CuO}$ nanoleaf syntheses $[18,21]$. These results suggest that never-dried $\mathrm{BC}$ is uniquely suited as a nanoscale scaffold or platform for nanomaterial deposition when compared to other cellulosic materials.

\section{Methods}

\subsection{Materials}

Yeast Extract, d-mannitol, and Citric Acid were purchased from Sigma-Aldrich (Sigma-Aldrich, St. Louis, MO, United States). Anhydrous Copper Sulfate, $\mathrm{NaOH}$ pellets, $\mathrm{Na}_{2} \mathrm{HPO}_{4}$, Peptone, and $28 \%$ Ammonia were purchased from Fisher Scientific (Thermo Fisher Scientific, Waltham, MA, USA). Pellicles of BC were collected from cultures of Gluconacetobacter hansenii (ATCC 23769, American Type Culture Collection, Manassas, VA, USA).

\subsection{Bacterial Cellulose Production}

The Gluconacetobacter hansenii bacteria were cultured in media with the following composition: $2 \%(w / v)$ d-mannitol, $0.5 \%(w / v)$ Yeast extract, $0.5 \%(w / v)$ Peptone, $0.27 \%(w / v) \mathrm{Na}_{2} \mathrm{HPO}_{4}$, and $0.125 \%$ $(w / v)$ Citric Acid. Bacteria were cultured in $20 \mathrm{~mL}$ of media in $85 \mathrm{~mm} \times 15 \mathrm{~mm}$, or $100 \mathrm{~mm} \times 15 \mathrm{~mm}$, in petri dishes for 4 days at $30^{\circ} \mathrm{C}$. After 4 days, pellicles were processed in $0.1 \mathrm{M} \mathrm{NaOH}$ at $\sim 95^{\circ} \mathrm{C}$ for $1 \mathrm{~h}$ to remove all bacteria and bacterial biofilm materials other than the $\mathrm{BC}$ matrix. Pellicles were then washed with reverse osmosis $(\mathrm{RO})$ water until a neutral $\mathrm{pH}$ was obtained. Pellicles were either used immediately or stored in $\mathrm{RO}$ water at room temperature. Pellicles in storage were boiled in $0.1 \mathrm{M}$ $\mathrm{NaOH}$ for $30 \mathrm{~min}$ and rinsed with $\mathrm{RO}$ water until at a neutral $\mathrm{pH}$ immediately prior to use.

\subsection{Characterization of $B C /$ Metal Oxide Nanocomposites}

The FTIR spectra were collected on an Agilent 670 FTIR Spectrometer (Agilent, Santa Clara, CA, USA) in attenuated total reflectance (ATR) mode. BC pellicles were air-dried and folded before FTIR analysis. Morphology of nanoparticles was analyzed via scanning electron microscopy. The scanning electron micrographs were obtained using a Zeiss Auriga FIB/FESEM with EDX (Zeiss, Jena, Germany). Scale bars were added using ImageJ software (U.S. National Institutes of Health, Bethesda, Maryland, USA). To measure the progress of $\mathrm{Cu}(\mathrm{OH})_{2}$ formation, we performed UV-Vis spectral analysis on an Agilent 6000i UV-Vis Spectrophotometer (Agilent, Santa Clara, CA, USA) with a spectrum obtained every $2 \mathrm{~min}$.

\section{4. $X R D$ Analysis}

The crystallinity of $\mathrm{BC}$ samples with and without metal oxide deposition were characterized using a Rigaku (Agilent) Gemini XRD (Rigaku, Tokyo, Japan). The samples were air-dried and then ground with a mortar and pestle. The powder was loaded into a borosilicate tube for analysis. For all XRD spectra, other than Figure 1B,C, a copper source was used at $40 \mathrm{~V}$ and $40 \mathrm{~mA}$.

For Figure 1F,H, a Molybdenum source at $40 \mathrm{~V}$ and $40 \mathrm{~mA}$ was used. The $2 \theta$ values of these spectra were then converted via $\mathrm{d}$ spacing to Copper 2 theta values by the following process. The equation 
$\mathrm{d}=\lambda /(2.0 \times \sin (0.5 \times 2 \theta \times \mathrm{D} 2 \mathrm{R}))$ was obtained from a U.S. Geological Survey website; with the wavelength of the $x$-rays $\lambda=1.5418 \AA$ which is a weighted average of Copper's $K \alpha 1$ and $K \alpha 2$, and $\mathrm{D} 2 \mathrm{R}=0.0174532925199433$ for converting between degrees and radians [22]. The above equation was solved for $2 \theta$ to give the following equation.

$$
2 \theta=\frac{\sin ^{-1}\left(\frac{\lambda}{2 d}\right)}{0.5 * D 2 R}
$$

The above equation was used to calculate a new $2 \theta$ value for each point on the Molybdenum spectra using d-values from the raw data file. To validate this method, a previously analyzed BC-CuO composite sample was analyzed via this process and the results were compared to a previously generated Copper source spectrum. The $\mathrm{CuO}$ and $\mathrm{BC}$ peak locations from the shifted spectrum agreed with peaks from the original Copper spectrum (Figure S4).

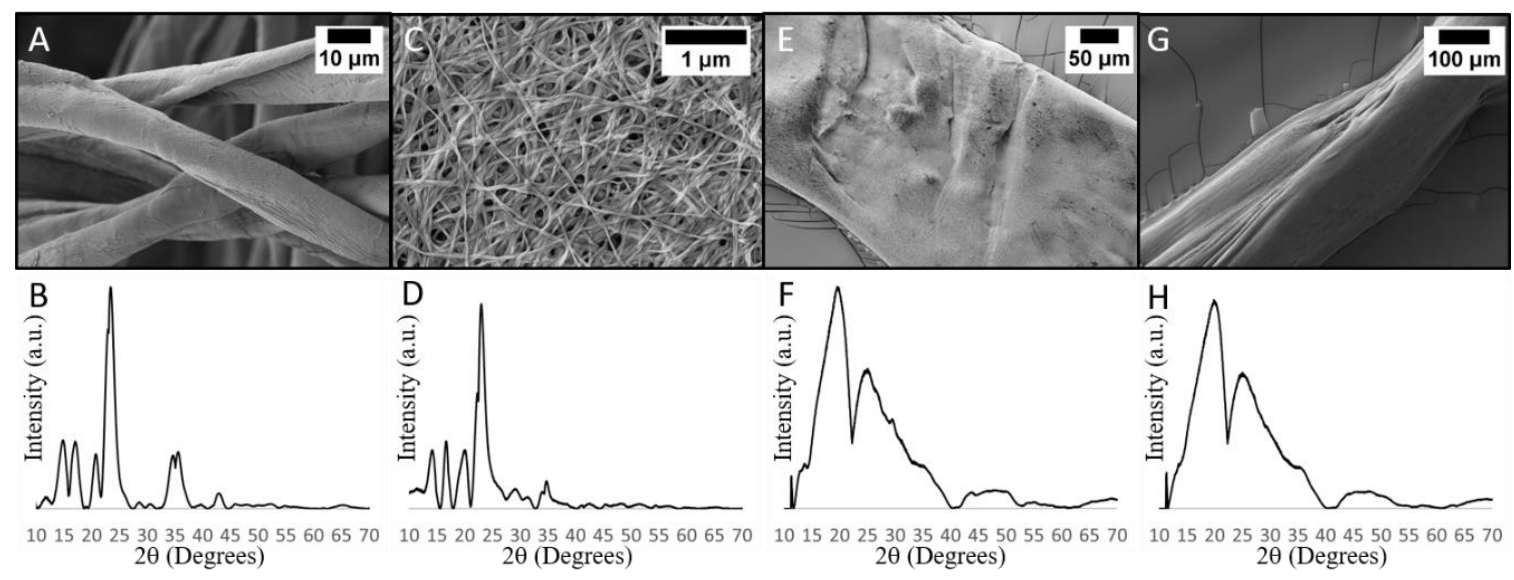

Figure 1. $\mathrm{CuO}$ deposition process performed on plain cotton, dried $\mathrm{BC}$, and regenerated cellulose fibers: SEM images (top) and XRD spectra (bottom) are shown for plain cotton (A,B), dried BC (C,D), regenerated cotton $(\mathbf{E}, \mathbf{F})$, and regenerated never-dried $B C(\mathbf{G}, \mathbf{H})$.

\subsection{Copper(II)Oxide Composite Formation}

Pellicles were soaked in $50 \mathrm{mM} \mathrm{CuSO}_{4}$ for $1 \mathrm{~h}$ and then rinsed with $\mathrm{RO}$ water 3-5 times. Rinsed pellicles were then added to $0.1 \mathrm{M} \mathrm{NaOH}$ for $5 \mathrm{~min}$ and then transferred to $0.1 \mathrm{M} \mathrm{NaOH}$ at $\sim 60^{\circ} \mathrm{C}$ for $30 \mathrm{~min}$. The $\mathrm{BC}-\mathrm{CuO}$ composite was rinsed until at a neutral $\mathrm{pH}$ and stored in water or dried for analysis.

\subsection{Regenerated Cellulose Fibers}

The following procedure is a modified protocol from a video on the NileRed Youtube channel which demonstrates how to dissolve and regenerate cellulose using Schweizer's reagent as the solvent [23-26].

To make the Schweizer's reagent, $100 \mathrm{~mL}$ of a $50 \mathrm{mM} \mathrm{CuSO}_{4}$ solution was made by dissolving anhydrous $\mathrm{CuSO}_{4}$ in $\mathrm{dH}_{2} \mathrm{O} .25 \mathrm{~mL}$ of $0.4 \mathrm{M} \mathrm{NaOH}$ was added to the $\mathrm{CuSO}_{4}$ solution to form $\mathrm{Cu}(\mathrm{OH})_{2}$. The solution was stirred and kept on ice until $\mathrm{Cu}(\mathrm{OH})_{2}$ formed. Moisture was removed from the $\mathrm{Cu}(\mathrm{OH})_{2}$ via vacuum filtration until a thick paste formed. The $\mathrm{Cu}(\mathrm{OH})_{2}$ paste was transferred to a graduated cylinder and $10 \mathrm{~mL}$ of $28 \%$ ammonia was added. The solution was stirred until the $\mathrm{Cu}(\mathrm{OH})_{2}$ dissolved completely and the solution took on a deep purple color.

Once the Schweizer's reagent was made, excess water was removed from never-dried BC, to prevent diluting the solution, by blotting on filter paper. Both cotton and never-dried BC were dissolved in batches until saturation and undissolved material was then removed. The now viscous solution was drawn into a luer-lok syringe, a 23G needle was attached, and the solution was ejected into a $10 \%$ sulfuric acid solution. For both never-dried BC and cotton, the regenerated fibers were 
formed by dissolution in Schweizer's reagent and subsequent injection into 10\% sulfuric acid [23-26]. The regenerated fibers were stirred in the $10 \%$ sulfuric acid until their blue color transitioned to opaque white. The regenerated fibers were rinsed until at a neutral $\mathrm{pH}$ and stored in $\mathrm{dH}_{2} \mathrm{O}$.

\section{Results and Discussion}

\subsection{CuO Nanoleaf Synthesis in a BC Matrix}

Before we can compare $\mathrm{BC}-\mathrm{CuO}$ synthesis to other cellulosic materials, we must first characterize our $\mathrm{BC}-\mathrm{CuO}$ nanocomposite. Typically, the first step in $\mathrm{CuO}$ synthesis involves the generation of $\mathrm{Cu}(\mathrm{OH})_{2}$ through the addition of $\mathrm{NaOH}$ to an aqueous solution that contains a copper salt and ammonia. $\mathrm{Cu}(\mathrm{OH})_{2}$ is a blue insoluble precipitate that, when heated, will transform via a dehydration and recrystallization reaction into a brown $\mathrm{CuO}$ precipitate $[20,27]$. The particle size, morphology, and crystallinity of the $\mathrm{CuO}$ nanomaterial is determined by reaction conditions and reagents [27-30]. For instance, the addition of increasing ammonia caused a transition from nanoplate to nanowire $\mathrm{CuO}$ particles [31]; while the addition of other amines to the reaction yields belts or leaves composed of $\mathrm{CuO}$ [32].

We adapted the common solution-based synthesis of $\mathrm{CuO}$ for the deposition of $\mathrm{CuO}$ onto the $\mathrm{BC}$ platform; however, this reaction only involves $\mathrm{CuSO}_{4}, \mathrm{NaOH}$, and a purified $\mathrm{BC}$ without additional capping and/or stabilizing components. In the first step of the in-situ $\mathrm{BC}$ mediated $\mathrm{CuO}$ deposition process; we incubated purified pellicles in $50 \mathrm{mM} \mathrm{CuSO}_{4}$ for $1 \mathrm{~h}$ and then removed excess $\mathrm{CuSO}_{4}$ through a series of extensive rinses with $\mathrm{RO}$ water. We then incubated the $\mathrm{Cu}^{2+}$ saturated $\mathrm{BC}$ pellicle in $0.1 \mathrm{M} \mathrm{NaOH}$, for $5 \mathrm{~min}$, which resulted in the immediate in-situ deposition of crystalline $\mathrm{Cu}(\mathrm{OH})_{2}$ within the $\mathrm{BC}$ matrix as indicated by XRD and the pellicle taking on a characteristic deep blue color (Figure 2D,F) [19]. We monitored the colorimetric reaction of the BC pellicle via time-lapse UV-Vis spectroscopy and observed an absorption peak at $640 \mathrm{~nm}$ after $4-6$ min post $0.1 \mathrm{M} \mathrm{NaOH}$ treatment (Supplemental Figure S1). SEM micrographs confirmed the deposition of nanomaterial with the presence of spiky depositions within the $\mathrm{BC}$ matrix (Figure $2 \mathrm{E}$ arrows). In the final step of this process, we heated the $\mathrm{BC}-\mathrm{Cu}(\mathrm{OH})_{2}$ pellicle to $60^{\circ} \mathrm{C}$ in $0.1 \mathrm{M} \mathrm{NaOH}$ for $30 \mathrm{~min}$. This resulted in the transformation of the $\mathrm{Cu}(\mathrm{OH})_{2}$ to $\mathrm{CuO}$, via a dehydration and recrystallization reaction, characterized by the $\mathrm{BC}$ pellicle turning brown (Figure 2G) [28]. SEM showed that the $\mathrm{CuO}$ had a nanoleaf morphology (Figure 2H) [33]. XRD analysis of the $\mathrm{BC}-\mathrm{CuO}$ material confirmed that the nanoleaves are indeed crystalline $\mathrm{CuO}$ particles as the $2 \theta$ peaks at $35.5^{\circ}$ and $38.5^{\circ}$ match the $[0,0,2]$ and $[1,1,1]$ crystal faces of $\mathrm{CuO}$, respectively (Figure 2I) [19]. The deposition of $\mathrm{CuO}$ onto the $\mathrm{BC}$ matrix was done under conditions that were faster, had a lower temperature, and without the use of capping reagents [20,27].

\subsection{Comparison of Never-Dried BC and Other Cellulosic Materials after $\mathrm{CuO}$ Deposition Process}

Recent research on $\mathrm{BC}$ nanocomposites has hypothesized that an interaction between metal ions and cellulose hydroxyl groups is responsible for nanomaterial deposition on $B C[11,13,18]$. To our knowledge, there has not been a study which further investigates this hypothesis. Since BC is chemically identical to other cellulosic materials, this hypothesis can be tested by performing the $\mathrm{CuO}$ deposition process shown in Figure 2 on other cellulosic materials. If the $\mathrm{CuO}$ deposition on never-dried $\mathrm{BC}$ is indeed facilitated by the adsorption of $\mathrm{Cu}^{2+}$ ions on cellulose hydroxyl groups, then we would also expect cotton and dried $\mathrm{BC}$ to facilitate $\mathrm{CuO}$ deposition. However, when we performed the $\mathrm{CuO}$ deposition process from Figure 2 on cotton, and dried $\mathrm{BC}$, we observed no $\mathrm{CuO}$ deposition on either material (Figure 1A-D).

The difference between never-dried BC and the cellulosic materials in Figure 1A-D is that never-dried $\mathrm{BC}$ has never been dehydrated. A whole never-dried BC pellicle has a thickness of a few millimeters and is comprised of $\sim 99 \%$ water. Upon drying, the pellicle undergoes an irreversible gel-to-film transition in which it shrinks to a thickness of less than $10 \mu \mathrm{m}[7,34]$. Since cellulose readily dries out when exposed to air, the BC must be stored in water to retain its never-dried state. 
Furthermore, in a process which has parallels to BC's gel-to-film transition, cellulose in the form of kraft wood pulp undergoes a largely irreversible loss of water in a process called hornification. Briefly, hornification is a process in which pores close and cellulose fibers bond with each other when no longer separated by water. This process takes place both within and between cellulose fibers as the individual cellulose polymers bond with each other during pore closure. This process affects the hydroxyl groups of cellulose as hydrogen bonds are thought to play a major role in hornification [35,36]. One possible explanation for the lack of deposition on dried $\mathrm{BC}$ and cotton is that the hydroxyl groups are simply not accessible to $\mathrm{Cu}^{2+}$ and $\mathrm{SO}_{4}{ }^{2-}$ ions due to inter- and intramolecular hydrogen bonding which occurs during drying.

Regenerated cotton fibers formed by Schweizer's reagent (i.e., cuprammonium rayon) have been shown to possess a large number of accessible hydroxyl groups [37]. If accessible hydroxyl groups in never-dried $\mathrm{BC}$ facilitate $\mathrm{CuO}$ deposition, then regenerated cotton should show $\mathrm{CuO}$ deposition since it has accessible hydroxyl groups. However, when we perform the $\mathrm{CuO}$ deposition process, outlined in Figure 2, on regenerated cotton fibers we see the fibers have the expected cellulose II crystal structure but fail to find any $\mathrm{CuO}$ deposition (Figure 1E,F). The lack of $\mathrm{CuO}$ deposition on regenerated cotton fibers suggests that never-dried $\mathrm{BC}^{\prime} \mathrm{s} \mathrm{CuO}$ deposition is not solely facilitated by accessible hydroxyl groups.

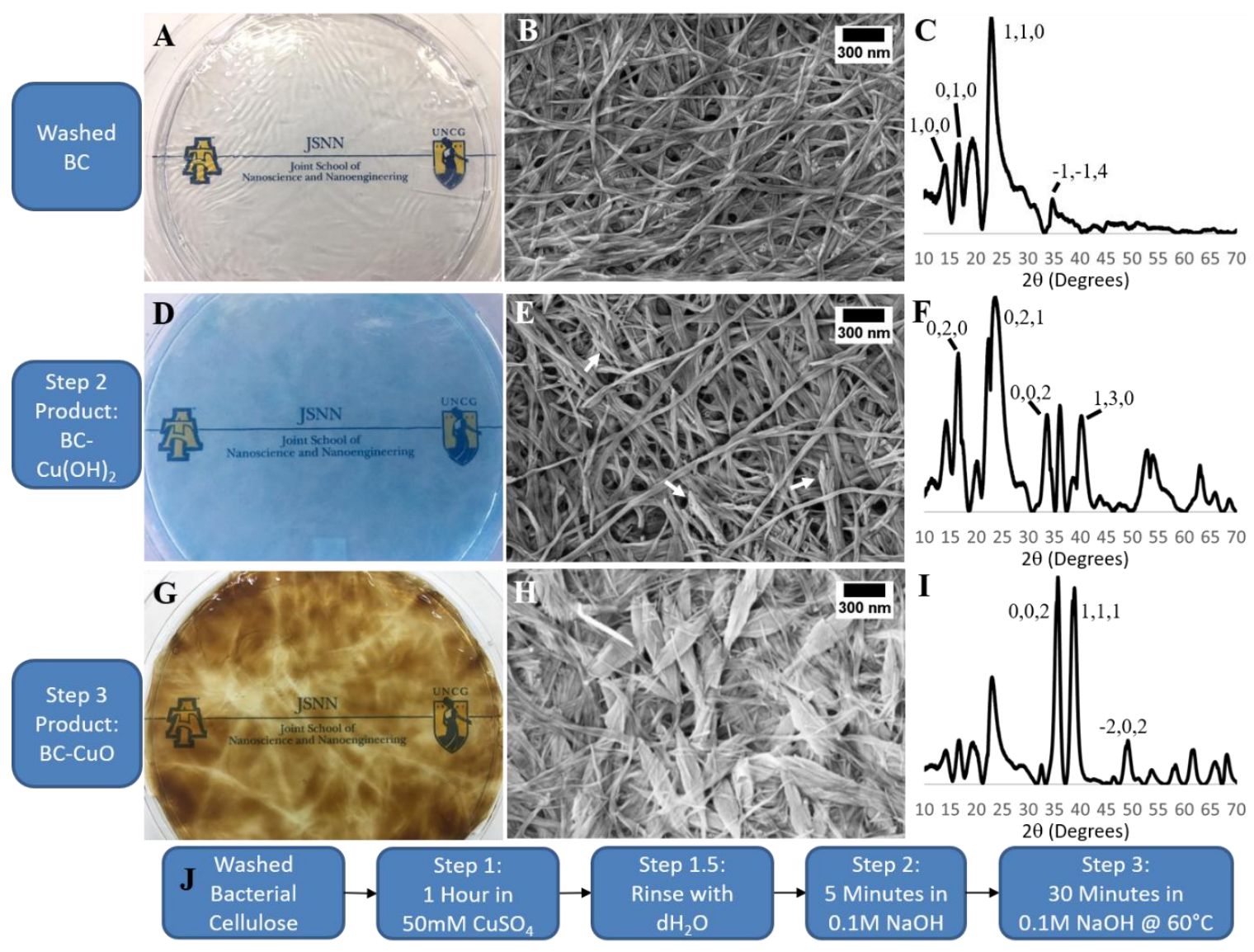

Figure 2. $\mathrm{BC}, \mathrm{BC}-\mathrm{Cu}(\mathrm{OH})_{2}$, and $\mathrm{BC}-\mathrm{CuO}$ Nanoleaf Composite: (A-C) Plain BC; (A) purified, never-dried BC pellicle demonstrating clear, colorless material, (B) SEM of a BC pellicle, (C) XRD of a BC pellicle; cellulose $\mathrm{I} \alpha$ peaks indicated. (D-F) In-situ formation of $\mathrm{Cu}(\mathrm{OH})_{2}$ in $\mathrm{BC}$ matrix; (D) never-dried BC pellicle after $\mathrm{Cu}(\mathrm{OH})_{2}$ deposition, (E) SEM of the $\mathrm{BC}-\mathrm{Cu}(\mathrm{OH})_{2}$ nanocomposite with spikes of $\mathrm{Cu}(\mathrm{OH})_{2}$ indicated (arrows), (F) XRD of $\mathrm{BC}-\mathrm{Cu}(\mathrm{OH})_{2}$ nanocomposite; $\mathrm{Cu}(\mathrm{OH})_{2}$ peaks indicated. (G-I) BC-CuO nanoleaf composite; (G) never-dried $\mathrm{BC}-\mathrm{CuO}$ pellicle, (H) SEM of BC-CuO nanoleaf composite, (I) XRD of $\mathrm{BC}-\mathrm{CuO}$ nanoleaf composite; $\mathrm{CuO}$ peaks indicated; (J) Outline of the $\mathrm{CuO}$ deposition process we performed. 


\subsection{Bacterial Cellulose Fiber Organization as a Mechanism for $\mathrm{CuSO}_{4}$ Retention during Rinsing}

The lack of $\mathrm{CuO}$ deposition in on rinsed regenerated cotton fibers (Figure 1E,F) warrants further discussion of cellulose structure in never-dried BC. Each BC nanofiber is a collection of smaller crystalline cellulose fibers. The smaller fibers are extruded from bacterial pores, then aggregated, and bond together in the extracellular space $[38,39]$. The smaller fibers do not completely bond as evidenced by neutron scattering research that showed never-dried BC nanofibers are nanoporous fibers through which water can diffuse [40].

As previously mentioned, when we subjected regenerated cotton fibers to our $\mathrm{CuO}$ deposition process, we did not observe $\mathrm{CuO}$ deposition; however, when the regenerated cotton was not rinsed after being soaked in $\mathrm{CuSO}_{4}$ we did observe significant $\mathrm{CuO}$ deposition (Figure S2). This result is similar to previous research which proposed that intrafibrillar pores in regenerated cellulose fibers can serve as spaces for $\mathrm{Fe}_{2} \mathrm{O}_{3}$ formation [41]. The key difference between performing $\mathrm{CuO}$ deposition on regenerated cotton and never-dried $\mathrm{BC}$ is that rinsing removes most, if not all, adsorbed $\mathrm{Cu}^{2+}$ and $\mathrm{SO}_{4}{ }^{2-}$ ions from regenerated cotton's intrafibrillar pores. Conversely, never-dried $\mathrm{BC}$ nanofibers retain some $\mathrm{Cu}^{2+}$ and $\mathrm{SO}_{4}{ }^{2-}$ ions after being rinsed, which can then be reacted to deposit $\mathrm{CuO}$.

Given that both never-dried BC and regenerated cotton fibers are fibers with intrafibrillar pores, the biggest difference between them is the fiber diameter. Dehydrated BC nanofibers are $\sim 40 \mathrm{~nm}$ in diameter while our regenerated cotton fibers have a diameter of at least $50 \mu \mathrm{m}$ when dehydrated. It is also important to note that the regenerated cotton fibers were dried before SEM imaging, and the fibers were significantly larger prior to drying. The massive difference in diameter suggests that the intrafibrillar pores of our regenerated cotton fibers are much larger than those of never-dried BC nanofibers. We hypothesize that during rinsing, due to never-dried $B C^{\prime}$ 's much smaller intrafibrillar pore size relative to regenerated cotton, water only removes $\mathrm{Cu}^{2+}$ and $\mathrm{SO}_{4}{ }^{2-}$ ions from the interfibrillar pore space and does not penetrate never-dried $\mathrm{BC}^{\prime} \mathrm{s}$ intrafibrillar nanopores. This process leaves behind $\mathrm{Cu}^{2+}$ and $\mathrm{SO}_{4}{ }^{2-}$ ions in the intrafibrillar pore space of never-dried $\mathrm{BC}$ which then deposits as $\mathrm{CuO}$.

If our intrafibrillar pore size hypothesis is correct, we would expect that fibers made of regenerated never-dried $B C$ would behave the same as regenerated cotton since the nanopores in never-dried $\mathrm{BC}$ would be destroyed during dissolution. We employed the previously mentioned Schweizer's reagent process to make regenerated cellulose fibers from never-dried $\mathrm{BC}$. These regenerated fibers were not dried and kept in water prior to testing. When we performed the $\mathrm{CuO}$ deposition process, outlined in Figure 2, on regenerated fibers made from never-dried $\mathrm{BC}$, we saw no $\mathrm{CuO}$ deposition (Figure $1 \mathrm{G}, \mathrm{H}$ ). This result demonstrates that the retention of $\mathrm{Cu}^{2+}$ and $\mathrm{SO}_{4}{ }^{2-}$ ions during rinsing is not simply a property intrinsic to never-dried $\mathrm{BC}$ and reinforces our hypothesis that fiber organization is responsible for the different responses displayed by the cellulosic materials we examined.

\subsection{When is Never-Dried BC Needed during BC-CuO Synthesis?}

To further examine the role of intrafibrillar nanopores during $\mathrm{CuO}$ deposition we performed the in-situ $\mathrm{BC}$ mediated $\mathrm{CuO}$ deposition, shown in Figure 2, on $\mathrm{BC}$ pellicles dried before different steps in the process. By drying out the $\mathrm{BC}$ before each step in the $\mathrm{CuO}$ deposition process we can gain insight into the role played by BC's intrafibrillar nanopores because the pores close when $B C$ is dried. The results of this experiment are shown below in Figure 3. 
A.
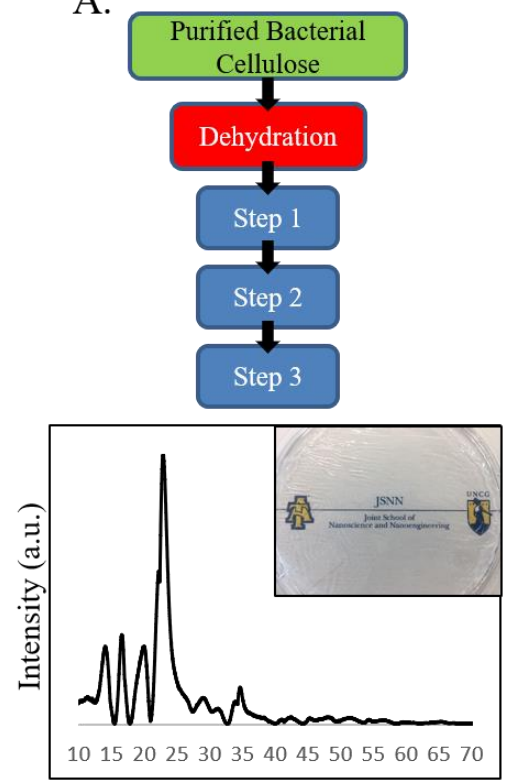

$2 \theta$ (Degrees)
B.

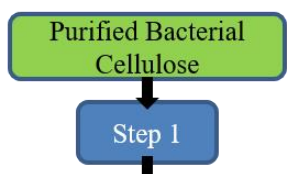

Dehydration

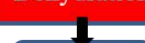

Step 2

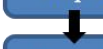

Step 3

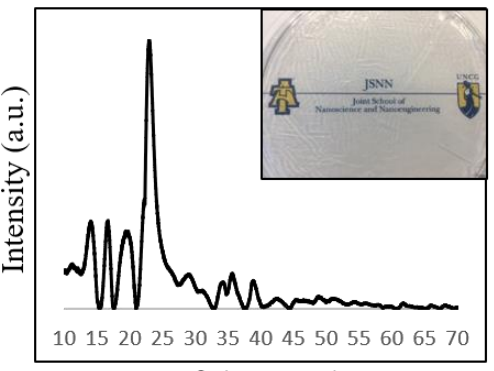

$2 \theta$ (Degrees)
C.

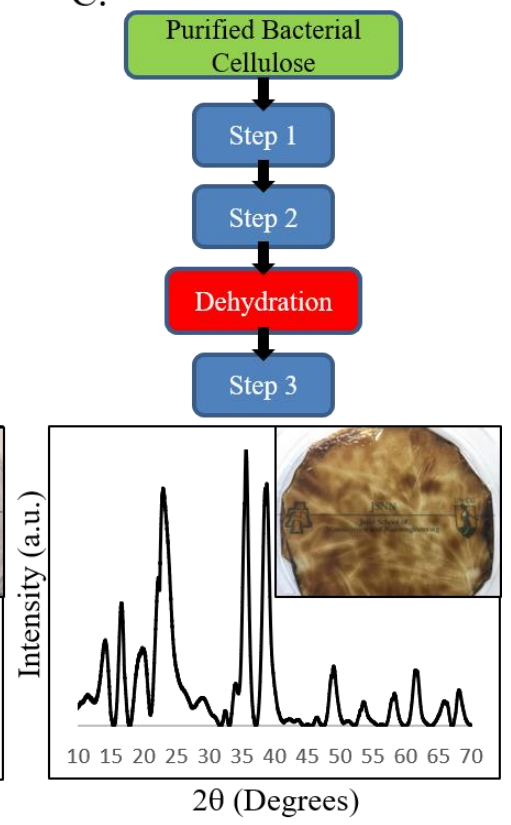

Figure 3. Hydration state of $\mathrm{BC}$ is essential for formation of $\mathrm{CuO}$ nanomaterial: Step 1, incubation of purified BC pellicle in $50 \mathrm{mM} \mathrm{CuSO}_{4}$; Step 2, formation of $\mathrm{BC}-\mathrm{Cu}(\mathrm{OH})_{2}$ composite material via incubation of the rinsed copper treated $B C$ pellicle in $0.1 \mathrm{M} \mathrm{NaOH}$; Step 3, heating $\mathrm{BC}-\mathrm{Cu}(\mathrm{OH})_{2}$ composite at $60{ }^{\circ} \mathrm{C}$ for $30 \mathrm{~min}$. (A) Dehydration of $\mathrm{BC}$ pellicle prior to Step 1 results in no formation of $\mathrm{CuO}$ material as evidenced by the $\mathrm{XRD}$ spectrum lacking the $\mathrm{CuO}$ signature and a pellicle with no color change (inset); (B) Dehydration of BC pellicle after copper treatment (Step 1) but before the formation of $\mathrm{Cu}(\mathrm{OH})_{2}$ (Step 2) also results in the failure to form $\mathrm{CuO}$ as evidenced by XRD and a lack of color change in the pellicle (inset); (C) Dehydration of the BC pellicle after $\mathrm{Cu}(\mathrm{OH})_{2}$ formation (Step 2) and prior to the heating (Step 3) results in the deposition of $\mathrm{CuO}$ material in the $\mathrm{BC}$ matrix, as evidenced by $\mathrm{XRD}$ and a brown colorimetric change to the pellicle (inset).

When the pellicle was dried prior to $\mathrm{CuSO}_{4}$ incubation (dried prior to Step 1, Figure 3A) the BC was in the same dehydrated state as cotton. Performing the $\mathrm{CuO}$ deposition process on dried $\mathrm{BC}$ resulted in no colorimetric change of the pellicle and no $\mathrm{CuO}$ XRD signature. This is the same result shown in Figure 1C,D. Drying after $\mathrm{CuSO}_{4}$ incubation and prior to $\mathrm{NaOH}$ incubation (dried prior to Step 2) resulted in very little $\mathrm{CuO}$ deposition. This is indicated by the lack of any colorimetric change in the pellicle and the lack of a strong $\mathrm{CuO}$ XRD signature (Figure 3B). This result is not surprising when viewed in the context of intrafibrillar pore closure. If intrafibrillar pores, which close during drying, contain $\mathrm{Cu}^{2+}$ and $\mathrm{SO}_{4}{ }^{2-}$ ions, then we would expect the ions to be expelled when the pores close. The expelled $\mathrm{Cu}^{2+}$ and $\mathrm{SO}_{4}{ }^{2-}$ ions then recombine and form the same $\mathrm{CuSO}_{4}$ hydrate that forms when the water in $\mathrm{CuSO}_{4}$ solution is allowed to evaporate (Figure S3). The water soluble $\mathrm{CuSO}_{4}$ Hydrate is removed during subsequent rinsing which results in very little $\mathrm{CuO}$ deposition. Finally, when the pellicle was not dried until after step 2 (dried prior to Step 3, Figure 3C), we observed a colorimetric change and $\mathrm{XRD}$ signature which closely resembles the $\mathrm{BC}-\mathrm{CuO}$ nanocomposite shown in the bottom row of Figure 2.

Based on the results of Figure 3, the association between $\mathrm{Cu}(\mathrm{OH})_{2}$ and the $\mathrm{BC}$ nanofibers is preserved during drying and subsequent rinsing which allows $\mathrm{CuO}$ to form during Step 3 of the process. However, an association between $\mathrm{Cu}^{2+}$ ions and $\mathrm{BC}$ nanofibers was not established when $\mathrm{BC}$ is dried before soaking in $\mathrm{CuSO}_{4}$ and is not preserved when $\mathrm{BC}$ is dried after soaking. In this procedure, the $\mathrm{Cu}(\mathrm{OH})_{2}$, which forms during Step 2, is only deposited if an association between $\mathrm{Cu}^{2+}$ ions and $\mathrm{BC}$ nanofibers is established during Step 1 and maintained during rinsing between Steps 1 and 2 (Step 1.5). This result further supports our hypothesis that the intrafibrillar nanopores of 
never-dried $\mathrm{BC}$ facilitates the retention of $\mathrm{Cu}^{2+}$ ions during rinsing between steps 1 and 2 of the $\mathrm{CuO}$ deposition process. It is this feature that differentiates never-dried BC from other cellulosic materials, and we hypothesize that it is due to the nanoscale size of its intrafibrillar pores.

\subsection{CuO Integration into the BC Matrix}

Unlike solution phase $\mathrm{CuO}$ nanomaterial synthesis, the $\mathrm{CuO}$ nanoleaves generated by this method were stably integrated in the $\mathrm{BC}$ matrix and resist removal even with significant agitation. Also, when never-dried $\mathrm{BC}$ pellicles were not thoroughly rinsed after soaking in $\mathrm{CuSO}_{4}$ a greater overall amount of $\mathrm{CuO}$ was deposited (Figure $4 \mathrm{C}$ ). Without thorough rinsing, much more $\mathrm{CuO}$ was not stably integrated in the $\mathrm{BC}$ matrix and precipitated into solution. This is apparent when comparing the amount of free $\mathrm{CuO}$ precipitated by an unrinsed pellicle (Figure 4B) to that of a thoroughly rinsed pellicle (Figure $4 \mathrm{E}$ ). This result further reinforces that there are at least two levels of arrangement of the $\mathrm{Cu}^{2+}$ ions within the $\mathrm{BC}$ matrix: one in which the $\mathrm{Cu}^{2+}$ ions are stability associated with the $\mathrm{BC}$ nanofibers and a bulk phase in which the $\mathrm{Cu}^{2+}$ ions are not associated with the $\mathrm{BC}$ nanofibers.
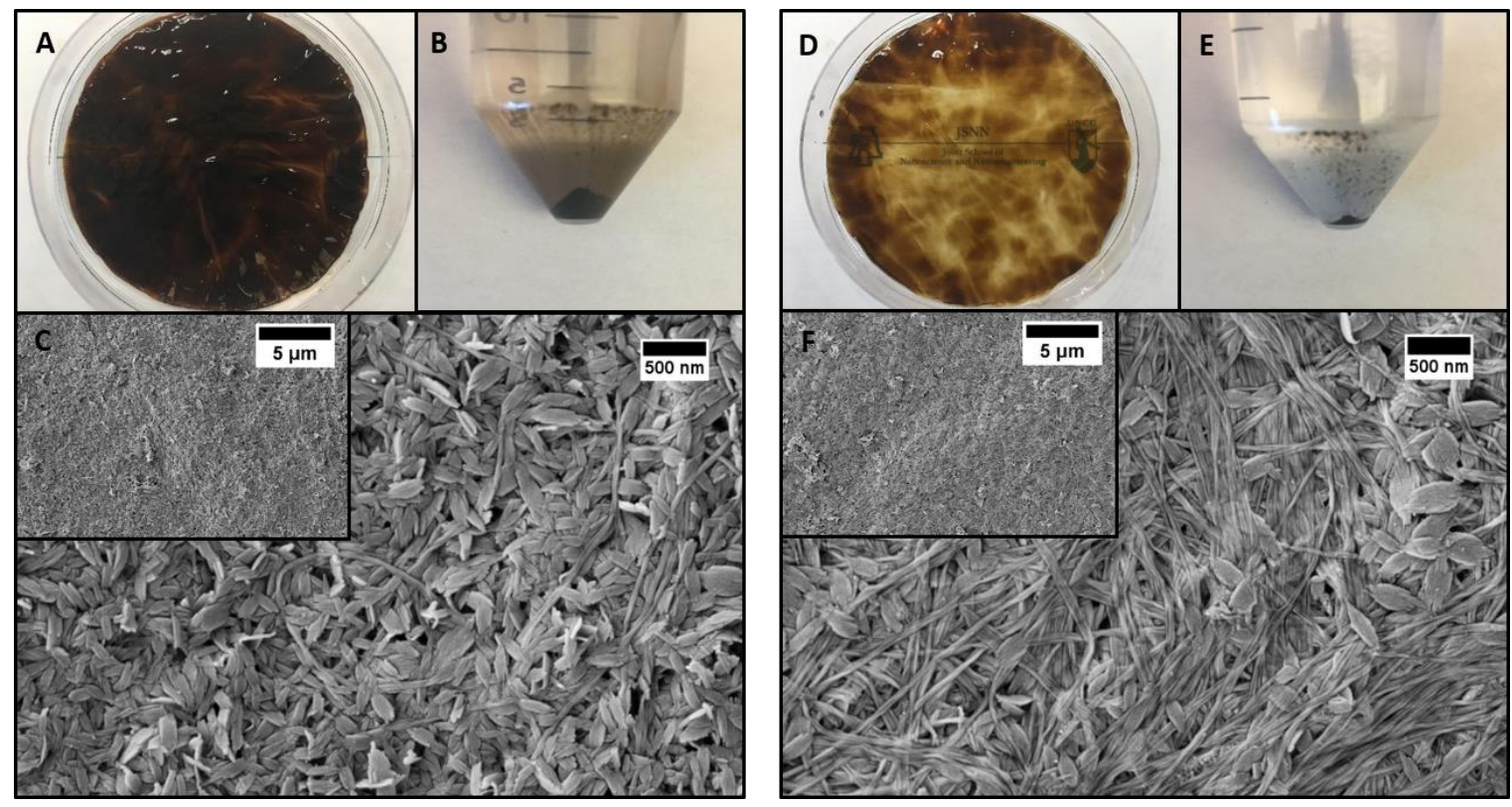

Figure 4. Removal of non-adsorbed $\mathrm{CuSO}_{4}$ via thorough rinsing: $(\mathrm{A}-\mathrm{C}) \mathrm{BC}-\mathrm{CuO}$ with no rinse after $1 \mathrm{~h}$ in $50 \mathrm{mM} \mathrm{CuSO}_{4}$ (no rinse after step 1); (A) Picture of BC-CuO, (B) Picture of pellet after centrifugation of step 3 reaction solution at $\sim 1750$ RCF for $10 \mathrm{~min},($ C) SEM of BC-CuO. (D-F) BC-CuO with thorough rinsing after $1 \mathrm{~h}$ in $50 \mathrm{mM} \mathrm{CuSO}_{4}$ (with rinse after step 1); (D) Picture of $\mathrm{BC}-\mathrm{CuO}$, (E) Picture of pellet after centrifugation of step 3 reaction solution at 1750 RCF for $10 \mathrm{~min}$, (F) SEM of BC-CuO.

The deposition of $\mathrm{CuO}$ is clearly non-uniform on the scale of an entire $\mathrm{BC}$ pellicle (Figure 4A,D). This brings up questions as to how non-uniform nanomaterial deposition could ultimately affect material performance. One cause of non-uniform deposition is how the $\mathrm{BC}$ is handled during processing. In our experiments, the $\mathrm{Cu}^{2+}$ loaded $\mathrm{BC}$ pellicles were removed from solution by a handheld sieve. This sieve caused the $\mathrm{Cu}^{2+}$ loaded pellicle to become folded and this could cause $\mathrm{BC}$ to expel $\mathrm{Cu}^{2+}$ ions at the creases. Another potential cause of non-uniformity is the $\mathrm{BC}$ itself. $\mathrm{BC}$ pellicles are not uniform materials because they are generated by free floating bacteria in media. This will inevitably lead to a non-uniform $\mathrm{BC}$ density across the pellicle on the nanoscale. However, depending on the application, other properties such as weight percentage could be more important than uniformity (i.e., photocatalysis). Furthermore, the ability of never-dried $\mathrm{BC}$ to retain some adsorbed $\mathrm{CuSO}_{4}$ after rinsing allows for further control over bulk nanocomposite properties. These concerns are 
highly application specific, and research will be needed to tailor BC nanocomposite synthesis to each application's needs.

\section{Conclusions}

In this paper, we demonstrated that $\mathrm{BC}$ facilitates the formation of $\mathrm{CuO}$ nanoleaves that are intimately integrated into the mechanically stable $\mathrm{BC}$ nanofiber matrix. The deposition reaction takes place under lower temperature conditions and with environmentally friendly reagents. We also provide a detailed characterization of $\mathrm{CuO}$ nanoleaf deposition into the $\mathrm{BC}$ matrix. In this paper we show that having accessible, hydrated, hydroxyl groups is not enough to explain why we observed $\mathrm{CuO}$ deposition on never-dried BC and not on the other cellulosic materials we tested. We propose that the primary feature of never-dried $\mathrm{BC}$ that facilitates $\mathrm{CuO}$ deposition is the intrafibrillar nanopores in each $\mathrm{BC}$ nanofiber. The exact interaction of solutes with the nanopores and how processing methods affect these interactions are open areas of future research. These details will be critical for the large-scale production of $\mathrm{CuO}$ and other metal oxide materials that use $\mathrm{BC}$ or other cellulosic materials as the deposition substrate. Furthermore, other crystalline polymeric materials may facilitate similar reactions, and further understanding of never-dried BC will aid the identification of these polymers.

Supplementary Materials: The following are available online at http://www.mdpi.com/2073-4360/11/11/1762/s1, Figure S1: Time-lapse UV-Vis spectrum, Supplemental Figure S2: SEM Images of Regenerated Cotton Fibers with $\mathrm{CuO}$ deposition, Supplemental Figure S3: XRD characterization of Step 1 product after drying, Supplemental Figure S4: Demonstration of Molybdenum source 2-theta shift with BC-CuO composite.

Author Contributions: Conceptualization, W.R.W. and D.R.L.; Formal analysis, W.R.W.; Funding acquisition, D.R.L.; Investigation, W.R.W.; Methodology, W.R.W. and D.R.L.; Project administration, D.R.L.; Resources, D.R.L.; Supervision, D.R.L.; Validation, W.R.W.; Visualization, W.R.W. and D.R.L.; Writing-original draft, W.R.W. and D.R.L.; Writing—review and editing, W.R.W. and D.R.L.

Funding: This work was supported by NIH grant to Dennis LaJeunesse (1R15EB024921-01). This work was performed at the Joint School of Nanoscience and Nanoengineering (JSNN), a member of Southeastern Nanotechnology Infrastructure Corridor (SENIC) and National Nanotechnology Coordinated Infrastructure (NNCI), which is supported by the National Science Foundation (ECCS-1542174).

Conflicts of Interest: The authors declare no conflict of interest.

\section{References}

1. Klemm, D.; Heublein, B.; Fink, H.P.; Bohn, A. Cellulose: Fascinating Biopolymer and Sustainable Raw Material. Angew. Chem. Int. Ed. 2005, 44, 3358-3393. [CrossRef] [PubMed]

2. Habibi, Y. Key Advances in the Chemical Modification of Nanocelluloses. Chem. Soc. Rev. 2014, 43, 1519-1542. [CrossRef] [PubMed]

3. Lee, K.Y.; Buldum, G.; Mantalaris, A.; Bismarck, A. More than Meets the Eye in Bacterial Cellulose: Biosynthesis, Bioprocessing, and Applications in Advanced Fiber Composites. Macromol. Biosci. 2014, 14, 10-32. [CrossRef] [PubMed]

4. Xue, Y.; Mou, Z.; Xiao, H. Nanocellulose as a Sustainable Biomass Material: Structure, Properties, Present Status and Future Prospects in Biomedical Applications. Nanoscale 2017, 9, 14758-14781. [CrossRef] [PubMed]

5. Klemm, D.; Kramer, F.; Moritz, S.; Lindström, T.; Ankerfors, M.; Gray, D.; Dorris, A. Nanocelluloses: A New Family of Nature-Based Materials. Angew. Chem. Int. Ed. 2011, 50, 5438-5466. [CrossRef] [PubMed]

6. Scott Williams, W.S.; Cannon, R.E. Alternative Environmental Roles for Cellulose Produced by Acetobacter Xylinum. Appl. Environ. Microbiol. 1989, 55, 2448-2452.

7. Fang, L.; Catchmark, J.M. Characterization of Water-Soluble Exopolysaccharides from Gluconacetobacter Xylinus and Their Impacts on Bacterial Cellulose Crystallization and Ribbon Assembly. Cellulose 2014, 21, 3965-3978. [CrossRef]

8. Florea, M.; Hagemann, H.; Santosa, G.; Abbott, J.; Micklem, C.N.; Spencer-Milnes, X.; de Arroyo Garcia, L.; Paschou, D.; Lazenbatt, C.; Kong, D.; et al. Engineering Control of Bacterial Cellulose Production Using a Genetic Toolkit and a New Cellulose-Producing Strain. Proc. Natl. Acad. Sci. USA 2016, 113, E3431-E3440. [CrossRef] 
9. Wiegand, C.; Elsner, P.; Hipler, U.C.; Klemm, D. Protease and ROS Activities Influenced by a Composite of Bacterial Cellulose and Collagen Type I in Vitro. Cellulose 2006, 13, 689-696. [CrossRef]

10. Ge, Y.; Chen, S.; Yang, J.; Wang, B.; Wang, H. Color-Tunable Luminescent CdTe Quantum Dots Membranes Based on Bacterial Cellulose (BC) and Application in Ion Detection. RSC Adv. 2015, 5, 55756-55761. [CrossRef]

11. Peng, S.; Fan, L.; Wei, C.; Liu, X.; Zhang, H.; Xu, W.; Xu, J. Flexible Polypyrrole/Copper Sulfide/Bacterial Cellulose Nanofibrous Composite Membranes as Supercapacitor Electrodes. Carbohydr. Polym. 2017, 157, 344-352. [CrossRef] [PubMed]

12. Yao, J.; Ji, P.; Wang, B.; Wang, H.; Chen, S. Color-Tunable Luminescent Macrofibers Based on CdTe QDs-Loaded Bacterial Cellulose Nanofibers for PH and Glucose Sensing. Sens. Actuators B 2018, 254, 110-119. [CrossRef]

13. Foresti, M.L.; Vazquez, A.; Boury, B. Applications of Bacterial Cellulose as Precursor of Carbon and Composites with Metal Oxide, Metal Sulfide and Metal Nanoparticles: A Review of Recent Advances. Carbohydr. Polym. 2017, 157, 447-467. [CrossRef] [PubMed]

14. Bin Hussein, M.Z.; Yahaya, A.H.; Ling, P.L.C.; Long, C.W. Acetobacter Xylenium as a Shape-Directing Agent for the Formation of Nano-, Micro-Sized Zinc Oxide. J. Mater. Sci. 2005, 40, 6325-6328. [CrossRef]

15. Chen, S.; Zhang, W.; Zhou, B.; Yin, N.; Wang, H.; Hu, W. Polyol Mediated Synthesis of ZnO Nanoparticles Templated by Bacterial Cellulose. Carbohydr. Polym. 2012, 92, 1953-1959. [CrossRef]

16. Sun, D.; Yang, J.; Wang, X. Bacterial Cellulose/TiO2 Hybrid Nanofibers Prepared by the Surface Hydrolysis Method with Molecular Precision. Nanoscale 2010, 2, 287-292. [CrossRef]

17. Wan, Y.; Yang, Z.; Xiong, G.; Luo, H. A General Strategy of Decorating 3D Carbon Nanofiber Aerogels Derived from Bacterial Cellulose with Nano-Fe3O4 for High-Performance Flexible and Binder-Free Lithium-Ion Battery Anodes. J. Mater. Chem. A 2015, 3, 15386-15393. [CrossRef]

18. Araújo, I.M.S.; Silva, R.R.; Pacheco, G.; Lustri, W.R.; Tercjak, A.; Gutierrez, J.; Júnior, J.R.S.; Azevedo, F.H.C.; Figuêredo, G.S.; Vega, M.L.; et al. Hydrothermal Synthesis of Bacterial Cellulose-Copper Oxide Nanocomposites and Evaluation of Their Antimicrobial Activity. Carbohydr. Polym. 2018, 179, 341-349. [CrossRef]

19. Pawar, S.M.; Pawar, B.S.; Inamdar, A.I.; Kim, J.; Jo, Y.; Cho, S.; Mali, S.S.; Hong, C.K.; Kwak, J.; Kim, H.; et al. In-Situ Synthesis of $\mathrm{Cu}(\mathrm{OH}) 2$ and $\mathrm{CuO}$ Nanowire Electrocatalysts for Methanol Electro-Oxidation. Mater. Lett. 2017, 187, 60-63. [CrossRef]

20. Shinde, S.K.; Fulari, V.J.; Kim, D.Y.; Maile, N.C.; Koli, R.R.; Dhaygude, H.D.; Ghodake, G.S. Chemical Synthesis of Flower-like Hybrid $\mathrm{Cu}(\mathrm{OH}) 2 / \mathrm{CuO}$ Electrode: Application of Polyvinyl Alcohol and Triton X-100 to Enhance Supercapacitor Performance. Colloids Surf. B Biointerfaces 2017, 156, 165-174. [CrossRef]

21. Sang, X.; Zhang, J.; Wu, T.; Zhang, B.; Ma, X.; Peng, L.; Han, B.; Kang, X.; Liu, C.; Yang, G. Room-Temperature Synthesis of Mesoporous $\mathrm{CuO}$ and Its Catalytic Activity for Cyclohexene Oxidation. RSC Adv. 2015, 5, 67168-67174. [CrossRef]

22. USGS Coastal and Marine Geology Program. Templates and Tables for Converting Degrees Two Theta to D-Spacings. Available online: https://pubs.usgs.gov/of/2001/of01-041/htmldocs/methods/2theta2d.htm (accessed on 2 April 2019).

23. NileRed. How to Dissolve Cotton in Water. Available online: https://www.youtube.com/watch?v= tvoimHLHdnA\&t (accessed on 2 April 2019).

24. Breslau, A. A Simplified Preparation of Schewitzer's Reagent. J. Chem. Educ. 1942, 19, 356. [CrossRef]

25. Wilson, W.K.; Launer, H.F. Preparing Cuprammonium Solvent and Cellulose Solutions. Anal. Chem. 1950, 22, 455-458. [CrossRef]

26. Burchard, W.; Habermann, N.; Klufers, P.; Serger, B.; Wilhelm, U. Cellulose in Schewizer's Reagent: A Stable, Polymeric Metal Complex with High Chain Stiffness. Angew. Chem. Int. Ed. 1994, 33, 884-887. [CrossRef]

27. Du, G.H.; Van Tendeloo, G. $\mathrm{Cu}(\mathrm{OH})_{2}$ Nanowires, $\mathrm{CuO}$ Nanowires and CuO Nanobelts. Chem. Phys. Lett. 2004, 393, 64-69. [CrossRef]

28. Cudennec, Y.; Lecerf, A. The Transformation of $\mathrm{Cu}(\mathrm{OH})_{2}$ into $\mathrm{CuO}$, Revisited. Solid State Sci. 2003, 5, 1471-1474. [CrossRef]

29. Ibupoto, Z.H.; Khun, K.; Lu, J.; Willander, M.; Ibupoto, Z.H.; Khun, K.; Lu, J.; Willander, M. The Synthesis of CuO Nanoleaves, Structural Characterization, and Their Glucose Sensing Application. Appl. Phys. Lett. 2013, 102, 103701. [CrossRef] 
30. Zhou, N.; Yuan, M.; Li, D.; Yang, D. One-Pot Fast Synthesis of Leaf-Like CuO Nanostructures and CuO/Ag Microspheres with Photocatalytic Application. Nano 2017, 12, 1750035. [CrossRef]

31. Li, Y.; Tan, B.; Wu, Y. Ammonia-Evaporation-Induced Synthetic Method for Metal (Cu, Zn, Cd, Ni) Hydroxide/Oxide Nanostructures. Chem. Mater. 2008, 20, 567-576. [CrossRef]

32. Zhao, Y.; Zhao, J.; Li, Y.; Ma, D.; Hou, S.; Li, L.; Hao, X.; Wang, Z. Room Temperature Synthesis of 2D CuO Nanoleaves in Aqueous Solution. Nanotechnology 2011, 22, 115604. [CrossRef]

33. Wang, X.; Yang, J.; Shi, L.; Gao, M. Surfactant-Free Synthesis of CuO with Controllable Morphologies and Enhanced Photocatalytic Property. Nanoscale Res. Lett. 2016, 11, 125. [CrossRef] [PubMed]

34. Cousins, S.K.; Brown, R.M. Cellulose-I Microfibril Assembly: Computational Molecular Mechanics Energy Analysis Favors Bonding by Vanderwaals Forces As the Initial Step in Crystallization. Polymer 1995, 36, 3885-3888. [CrossRef]

35. Déléris, I.; Wallecan, J. Relationship between Processing History and Functionality Recovery after Rehydration of Dried Cellulose-Based Suspensions: A Critical Review. Adv. Colloid Interface Sci. 2017, 246, 1-12. [CrossRef] [PubMed]

36. Pönni, R.; Vuorinen, T.; Kontturi, E. Proposed Nano-Scale Coalescence of Cellulose in Chemical Pulp Fibers during Technical Treatments. BioResources 2012, 7, 6077-6108. [CrossRef]

37. Yamane, C. Structure Formation of Regenerated Cellulose from Its Solution and Resultant Features of High Wettability: A Review. Nord. Pulp Paper Res. J. 2015, 30, 78-91. [CrossRef]

38. Torres, F.G.; Arroyo, J.J.; Troncoso, O.P. Bacterial Cellulose Nanocomposites: An All-Nano Type of Material. Mater. Sci. Eng. C 2019, 98, 1277-1293. [CrossRef]

39. Bogdanova, O.I.; Chvalun, S.N. Polysaccharide-Based Natural and Synthetic Nanocomposites. Polym. Sci. Ser. A 2016, 58, 629-658. [CrossRef]

40. Martínez-sanz, M.; Mikkelsen, D.; Flanagan, B.; Gidley, M.J.; Gilbert, E.P. Multi-Scale Model for the Hierarchical Architecture of Native Cellulose Hydrogels. Carbohydr. Polym. 2016, 147, 542-555. [CrossRef]

41. Sun, J.; Guan, J.; Xiang, J.; Zhang, L.; Zhou, J.; Liu, S. Fiberlike Fe2O3 Macroporous Nanomaterials Fabricated by Calcinating Regenerate Cellulose Composite Fibers. Chem. Mater. 2008, 20, 3623-3628. [CrossRef]

(C) 2019 by the authors. Licensee MDPI, Basel, Switzerland. This article is an open access article distributed under the terms and conditions of the Creative Commons Attribution (CC BY) license (http://creativecommons.org/licenses/by/4.0/). 